\title{
Socioeconomic influences on breastfeeding and weaning practices in a Mediterranean setting
}

\section{Abstract}

The World Health Organisation (2016) recommends exclusive breastfeeding up to 6 months of age and the introduction of complementary foods at around 6 months. International literature suggests that although knowledge of the guidelines is high, only a minority of parents wait until 6 months to wean and sub-optimal weaning practices are common. The aim of this study was to assess breastfeeding and weaning practices and to evaluate the effect of socio-economic determinants on such behaviours in a Mediterranean setting.

A cross-sectional study design was used. One-time short interviews with 250 consenting mothers having 8 month old infants were conducted using a structured questionnaire. Mothers attending all the well baby clinics in the primary health care centres of the Maltese Islands for a period of two months were included in the study. The questionnaire included socio-economic and demographic data, details on the initiation and duration of breastfeeding, weaning practices and a short assessment of the infant's usual diet at 8 months.

Mothers between 25-34 years old (26\%) and mothers with a high level of education (25\%) were more likely to breastfeed only during the first 8 months of life without using any other type of milk, compared to younger $(6.5 \%)$ or older $(21 \%)$ mothers, and to mothers with a lower level of education $(16 \%)$. The level of education was also positively associated with the continuation of breastfeeding, with the mean duration of breastfeeding for higher educated mothers being 4 months compared to 2.6 months in less educated mothers. Mothers with a lower level of education and mothers who are house-wives are more likely to give their infants only readymade foods however associations were in this case borderline significant. On the other hand, appropriateness of weaning age was not associated with any socio-economic factors.

The length of breastfeeding and the introduction of complementary foods varied widely between mothers. Compliance with infant feeding guidelines was poor. The mother's age, level of education and employment status all have an impact on breastfeeding and weaning practices. The provision of professional advice and a supportive environment for mothers, together with interventions for increasing awareness may promote maternal adherence to WHO guideline.

\section{Conflict of Interest}

There is no conflict of interest 